\title{
Biogeography in optimization algorithms: a closer look
}

\author{
Padarabinda Palai ${ }^{1}$, Debani Prashad Mishra ${ }^{2}$, Surender Reddy Salkuti ${ }^{3}$ \\ ${ }^{1}$ Department of Computer Science Engineering, IIIT Bhubaneswar, Odisha, India \\ ${ }^{2}$ Department of Electrical and Electronics Engineering, IIIT Bhubaneswar, Odisha, India \\ ${ }^{3}$ Department of Railroad and Electrical Engineering, Woosong University, Daejeon, Republic of Korea
}

\begin{tabular}{|c|c|}
\hline Article Info & ABSTRACT \\
\hline Article history: & Biogeography can be broken down into bio and geography, which would \\
\hline Received Jan 23, 2021 & $\begin{array}{l}\text { imply the geography, i.e., the dispersion of biological organisms. The entire } \\
\text { field of biology inspired algorithm is inclined towards providing the most }\end{array}$ \\
\hline Revised Sep 14, 2021 & optimal solution for a given problem set. Computer science experts want to \\
\hline Accepted Sep 25, 2021 & $\begin{array}{l}\text { always learn from the surroundings. Nature is sporadic and spontaneous and } \\
\text { the erratic nature of a habitat is the very differentiating factor between a real }\end{array}$ \\
\hline Keywords: & $\begin{array}{l}\text { world and an ideal world problem. Things change and that nothing remains } \\
\text { constant. The diversification of a certain habitat is bound to change through }\end{array}$ \\
\hline Biogeography & external influences, some for the better, and some for the worse. This paper \\
\hline Immigration rate & tries to mimic the natural influences in a habitat in a python environment and \\
\hline Migration & $\begin{array}{l}\text { try to come up with a minimal objective value atter iterating through the given } \\
\text { metaheuristic algorithm. }\end{array}$ \\
\hline
\end{tabular}

Nature inspired algorithms

Optimization

This is an open access article under the CC BY-SA license.

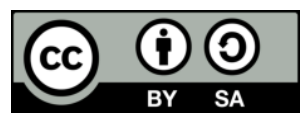

Corresponding Author:

Surender Reddy Salkuti

Department of Railroad and Electrical Engineering

Woosong University

17-2, Jayang-dong, Dong-gu, Daejeon-34606, Republic of Korea

Email: surender@wsu.ac.kr

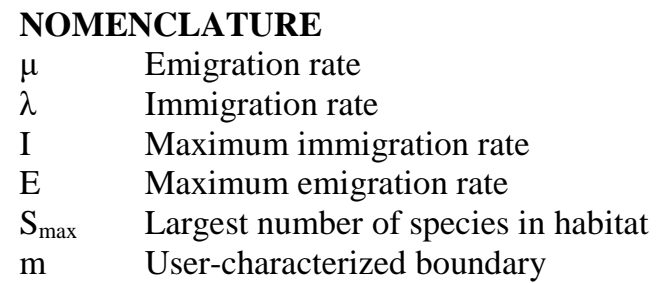

\section{INTRODUCTION}

Biogeography is a field that has been worked upon by naturalists in Charles Darwin and Wallace [1]. However not until recently the mathematical modelling of such a domain had not been worked on. In the $60 \mathrm{~s}$ scientists really looked into how the distribution of a certain island changed when there was an influx of individuals or outflow of masses due to disasters. The very aspect of extinction as well as migrating to nearby habitats also created a lot of interest for developing a mathematical model that would be efficient and less error prone [2]. However, science hadn't evolved a lot back then. Right now, things are different and we have access to practically any piece of science. Several challenges have been faced while trying to model the behaviour of the organisms in a specific habitat [3]. However with exponential advancements in technology and increasing computational advantages it has now become possible to optimize the problems people once thought were not feasible [4]. Biogeography when used in computer science has similar effects that other 
algorithms that are population based have had in the past [5]. It is a field that is still in a very nascent phase and obviously has a very large scope for research and development [6]. This paper tries to provide a very pragmatic and basic solution and tries to come up with a visual output of the function that has been minimized after repeated iterations in ideal conditions where the values are properly assumed [7].

To introduce the paper, two terminologies are very crucial, i.e., habitat suitability index (HSI) and suitability index variable (SIV) [8]. The former is dependent on the latter while the opposite doesn't hold true. SIV denotes the set of factors that affect a certain habitat like weather, temperature, humidity, precipitation rates, contrast in seasons, availability of food and water. These determine how good a certain habitat is [8]. Naturally it makes sense that habitats with a high habitat suitability index prosper more in comparison with habitats with very minimal habitat suitability index [9]. Where there is a very high value of HSI, there obviously more species and individuals would exist. The dispersion of species does not change and tends to reach a state of saturation [10]. The species in such habitats have high emigration rates and those with low HSI, tend to have high immigration rates [10]. The chances are really low with the species of such habitats. There are also chances if the species remain static in their dispersion rates and that they are likely to go extinct. But the high immigration rate ensures diversity in comparison with islands with high habitat suitability index. Again, the SIV values are crucial in determining the HSI values [11].

The objectives of this paper are twofold. To begin with, we need to give an overall introduction of the new metaheuristic algorithm in "biogeography-based optimization (BBO)" [12]. The plain biogeography is considered, computed and at that point summing it up to get a universally useful optimization calculation. Second, we need to thoroughly study the plots of the benchmarks of the BBO optimization. This paper has demonstrated how BBO, a research into the topographical dispersion of biological habitats, can be used to determine optimization algorithms. Such a set of algos give rise to biogeography based optimization. We have coded the entire algorithm in a python 3.8 virtual environment and have received values for user determined inputs for a habitat that has been coded assuming ideal conditions. The algorithm has been run 500 times and that the elitism parameter has been kept as 2 . The way this paper mimics the actual behaviour of a habitat and comes up with optimal solutions efficiently is something that is really commendable. This paper is fundamental and, consequently, provides massive scope of opportunities for additional exploration.

\section{RESEARCH METHOD}

This paper attempts to initiate the research into the topic of biogeography by first understanding the basics of a species model [13]. A species model provides a graphical overview as to how the species count of a certain habitat changes with inflow or the outflow due to external factors [14]. The graph as shown in Figure 1 shows the behaviour of immigration and emigration under ideal conditions [15]. However, things aren't ideal and the curves will change depending on circumstances [16]. However with the sole objective of understanding the concept, we assume that conditions are ideal. Figure 1 shows the species abundance in a single habitat.

The entire aspect of emigration is governed by the very aspect that more the number of individuals in a certain island, more is the dispersion in species and that higher is the opportunity of the population to emigrate off to a new island [17]. Now, let's discuss equilibrium. It is an ideal state where the lambda and mu values are the same and that the species distribution lying near this point has the possibility of dominating the data set [18]-[20]. It is denoted by $S_{0}$. This value will change positively and negatively with spurts of immigrating species and disastrous events like disease outbreak, ravenous predators respectively. After a major perturbation, nature will take a massive amount of decades to reach a point of stability (i.e., $\mathrm{S}_{0}$ ) [21]. Code for calculating emigration rate $(\mu)$ and immigration rate $(\lambda)$ is given below:

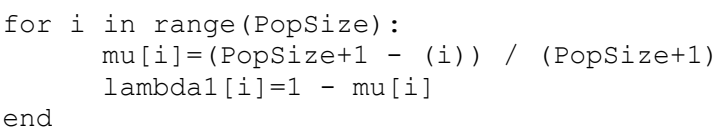

The probability in $\mathrm{P}_{\mathrm{s}}$ that a habitat contains $\mathrm{S}$ species in time $\mathrm{t}$ while the time changes with $\Delta \mathrm{t}$ is given by (1),

$$
P_{S}(t+\Delta t)=P_{S}(t)\left(1-\lambda_{s} \Delta t-\mu_{s} \Delta t\right)+P_{S}-1 \lambda_{s}-1 \Delta t+P_{S}+1 \mu_{s}+1 \Delta t
$$

The (1) is true for time change $(\Delta t)$ and one of the following condition must be satisfied:

- At initial time t, the number of species, i.e., $S$ did not change

- When one species immigrated, the number of species was $\mathrm{S}-1$

- When one species emigrated, the number of species was $\mathrm{S}+1$ 
The assumption in this work is $\Delta \mathrm{t}$ is so minimal so as to nullify emigration and immigration [22]. Taking the limit of aforementioned equation at $\Delta t \rightarrow 0$ is given by (2),

$$
P_{S}=\left\{\begin{array}{cl}
-\left(\lambda_{s}+\mu_{s}\right) P_{s}+\mu_{s+1} P_{s+1} & \text { for } S=0 \\
-\left(\lambda_{s}+\mu_{s}\right) P_{s}+\lambda_{s-1} P_{s-1}+\mu_{s+1} P_{s+1} & \text { for } 1 \leq S \leq\left(S_{\text {max }}-1\right) \\
-\left(\lambda_{s}+\mu_{s}\right) P_{s}+\lambda_{s-1} P_{s-1} & \text { for } S=S_{\text {max }}
\end{array}\right.
$$

Figure 2 depicts the species model, and from this figure, it is clear that $\mathrm{S}_{1}$ is a poorer solution in comparison to $S_{0}$. This is because the fitness of $S_{1}$ is significantly higher than that of $S_{0}$ and hence it would resist change to any of it's SIV values.

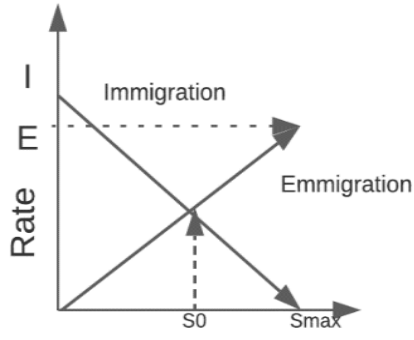

No of Species

Figure 1. Behaviour of immigration and emigration under ideal conditions

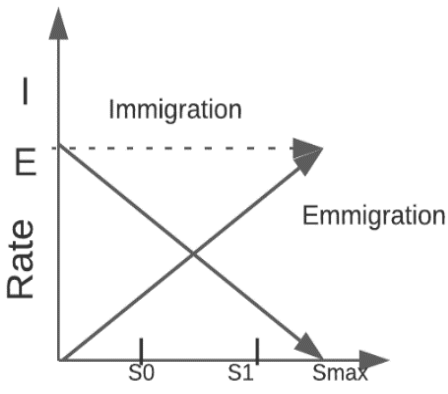

Figure 2. Species model

From the species model, one can determine,

$$
\begin{aligned}
& \mu_{k}=\frac{E k}{n} \\
& \lambda_{k}=I\left(1-\frac{k}{n}\right)
\end{aligned}
$$

now for the E=I (a special case), satisfies the following condition,

$$
\mu_{k}+\lambda_{k}=1
$$

\subsection{Migration}

Migration is a core concept of this paper. This paper has initialized the population of a habitat in terms of an array of integers in python programming with finite dimensions. Each solution index of the array can be considered an SIV. The array in the program is analogous to vectors of integers in the paper [23]. Now as mentioned previously the HSI value, often referred to as fitness, is a very important concept. The more the HSI value, the more is the emigration and less HSI means more immigration. The objective of this paper is to share the $\lambda$ and $\mu$ data between different solutions to optimize the algorithm [24], [25]. With probability $\mathrm{P}_{\mathrm{Mod}}$, we change each solution on the basis of others [26]. Say a certain iteration step is picked then we use the lambda to probabilistically decide if the suitability index variable is to be modified [27]. If a specific dimension of a solution is specified then we decide if the mu rate is to be applied to another solution whose SIV will be random.

Step 1: Initializing the population

\#Initializing Population with SIV values

pos=numpy . random uniform $(0,1,($ Popsize, dim) $) *(u b-1 b)+1 b$

\#Calculate objective function for each particle

for $i$ in range (Popsize):

\# Performing the bound checking

pos $[i,:]=$ numpy. $\operatorname{clip}(\operatorname{pos}[i,:]$, lb, ub)

fitness=objf (pos $[i,:])$

fit $[i]=f i t n e s s$

Int J Artif Intell, Vol. 10, No. 4, December 2021: 982 - 989 


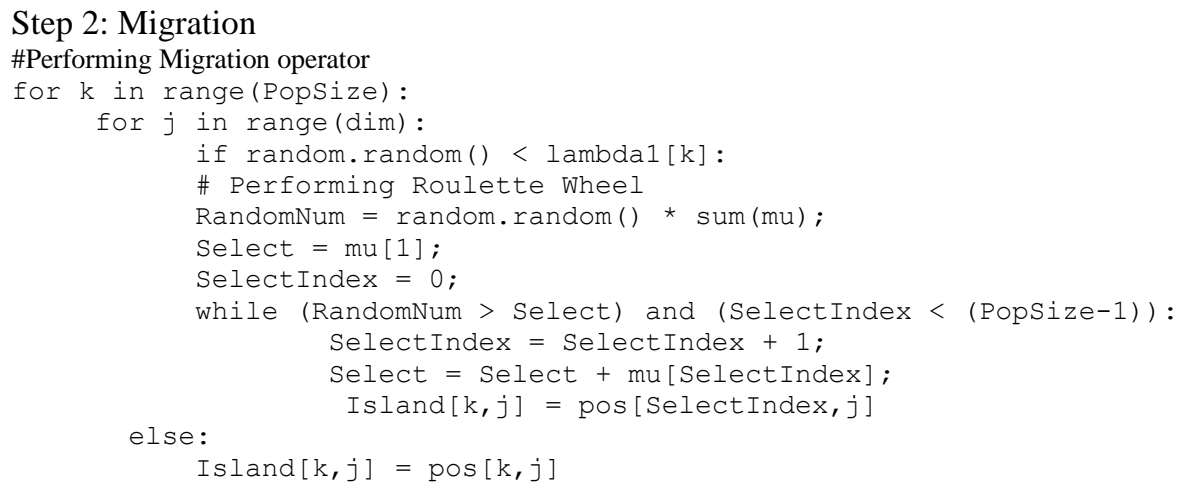

\subsection{Mutation}

Disastrous functions can radically change the habitat suitability index of a function domain i.e a habitat. What happens is that the population count diversifies and the fact that the entire act is inevitable makes mutation a very important aspect of biogeography. We use this algorithm to train a model on suitability index variable mutation and that population count is used to make sure of the mutation [28], [29].

$$
m(s)=m_{\max }\left(\frac{1-P_{s}}{P_{\max }}\right)
$$

This step will mainly disperse the dataset causing greater variance. With this it is also ensured that all solutions have an equal chance of being dominant in the search space. The (2) will govern the aforementioned step in mutating species [30], [31]. What happens is that high HSI and low HSI solutions are not probable because they do not lie in the middle of the curve shown in the species model. The solutions that lie in the middle i,e equilibrium are very much probable to exist. So the extremities have to mutate to exist and that is the law of nature.

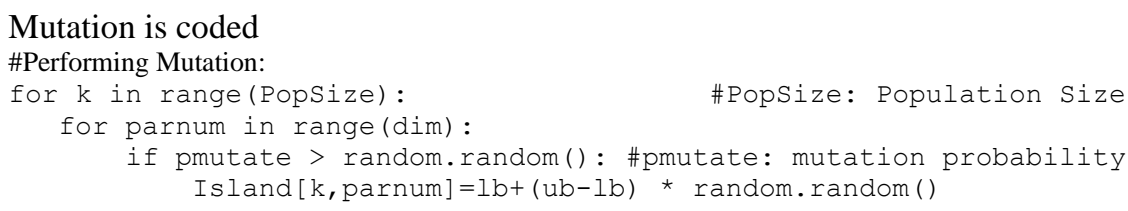

The steady state value for existence of each species is governed by (7),

$$
P(\infty)=\frac{v}{\sum_{i=1}^{n+1} v_{i}}
$$

where the value of $\mathrm{v}$ is equal to an eigen vector which has been defined as sets of arrays in the python models that this paper presents [32], [33]. The set of vectors are initialized in an uniform random distribution and only then the computation starts to ensure that the model is flexible in its approach in mimicking a natural habitat and the corresponding behaviour of the species existing in it.

\subsection{Algorithm}

The Biogeography based optimization algorithm by conmibing the migration and mutation phases has been presented next:

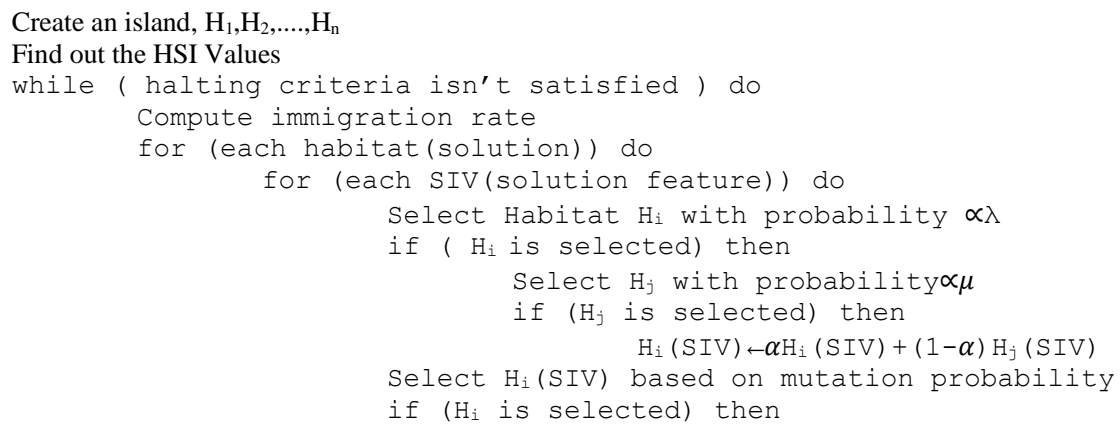


Recompute HSI value

Replace $H_{i}($ SIV) with random SIV

\section{RESULTS AND DISCUSSION}

This section compares BBO techniques against different algorithms that are based on population behavior [34]. This paper has displayed a set of tables that consists of norms of benchmarks that serve as a source of comparison with the biogeography based algorithms. and Then we display the entire project that was coded in Python 3.8 to implement the paper.

\subsection{Benchmark results}

To gauge the efficiency of this algorithm we execute it 100 times over 2 generations and observe the plot over various benchmark functions. The granularity of every single domain was 0.1 but the quartic function had 0.01 [35]. The functions provided in the table are commonly used in research works in PB algorithms as a standard comparison measure. Some have a single, unique minima, while some have multiple i.e., they are multimodal.Some can be differentiated at every point in the search space and some cannot be separated. Figure 3 shows that around the $17^{\text {th }}$ iteration there is a steep decline in the fitness values or a considerable increase in the convergence rate. BBO performs faster than GAs on this benchmark. Figure 3(a) shows that around the $17^{\text {th }}$ iteration there is a steep decline in the fitness values or a considerable increase in the convergence rate. BBO performs faster than GAs on this benchmark. Figure 3(b) shows that the BBO minimizes the values rapidly only after a few iterations. The convergence rate is very high for this benchmark and it gradually closes in on 0 after the $24^{\text {th }}$ iteration.

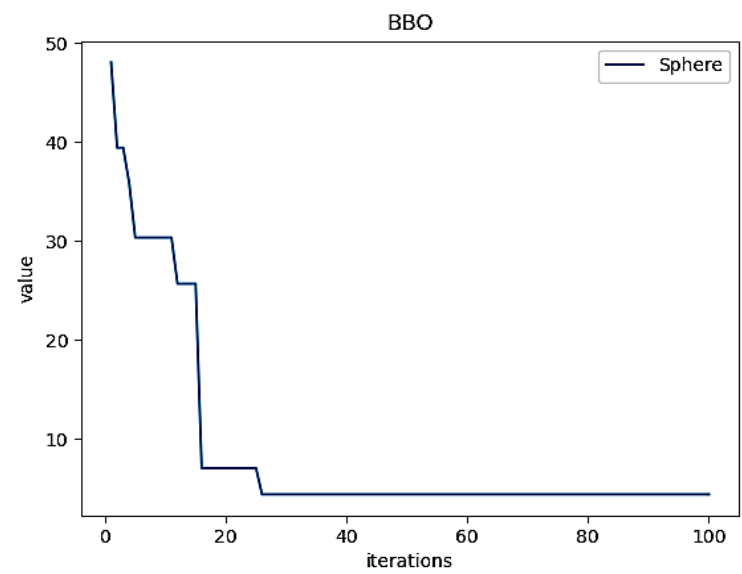

(a)

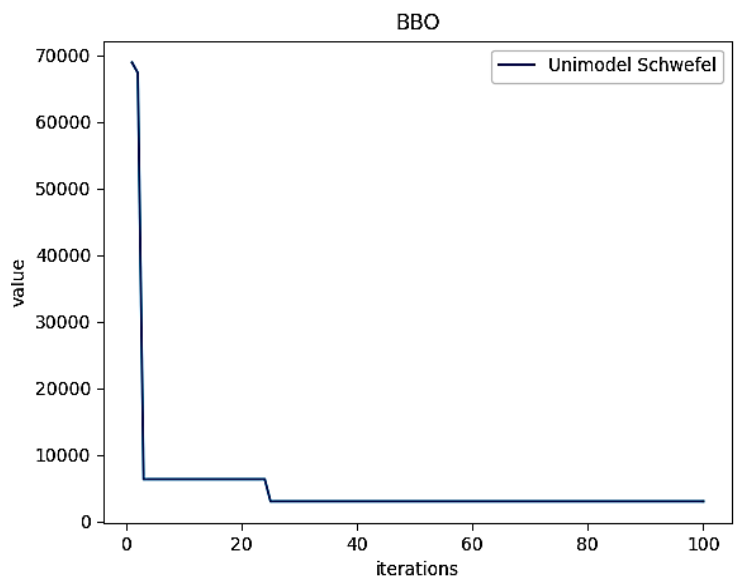

(b)

Figure 3. Plot of n-dimensional: (a) unimodal convex spherical benchmark, (b) unimodal continuous schwefel benchmark 
Figure 4(a) shows that on a multimodal function the steep convergence occurs on a couple of occasions. After a few iterations the fitness minimizes significantly and then around the $10^{\text {th }}$ iteration it converges to 0 . For rastrigin function as shown in Figure 4(b), the fitness value converges to 0 after gradual declinations on the $3^{\text {rd }}, 15^{\text {th }}, 38^{\text {th }}$ iteration. It's faster than the PSO on this benchmark.

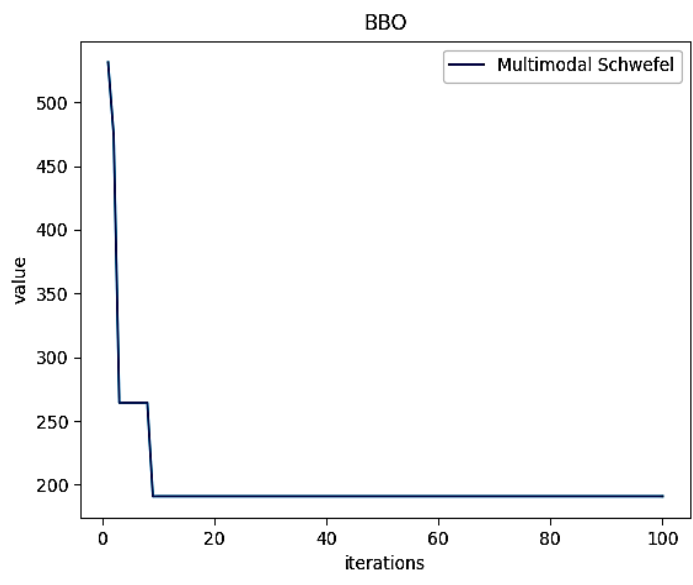

(a)

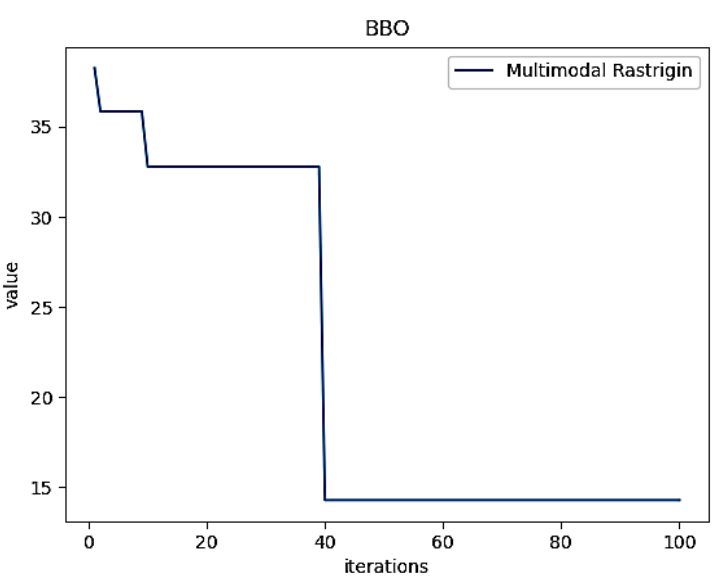

(b)

Figure 4. Plot of: (a) continuous non-convex multimodal schwefel benchmark, (b) multimodal n-dimensional convex multimodal rastrigin benchmark

In the Ackley benchmark the BBO has the fastest convergence rate and obtains the local minima fastest of all the population based algorithms and it is shown in Figure 5(a). Figure 5(b) is a combined plot of all the benchmarks. The $\mathrm{Y}$-axis denotes the fitness value and $\mathrm{X}$-axis denotes the number of iterations.

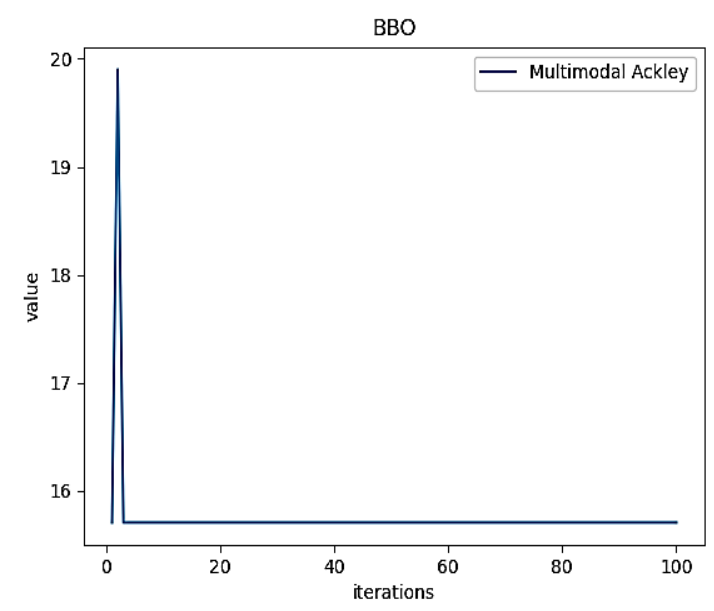

(a)

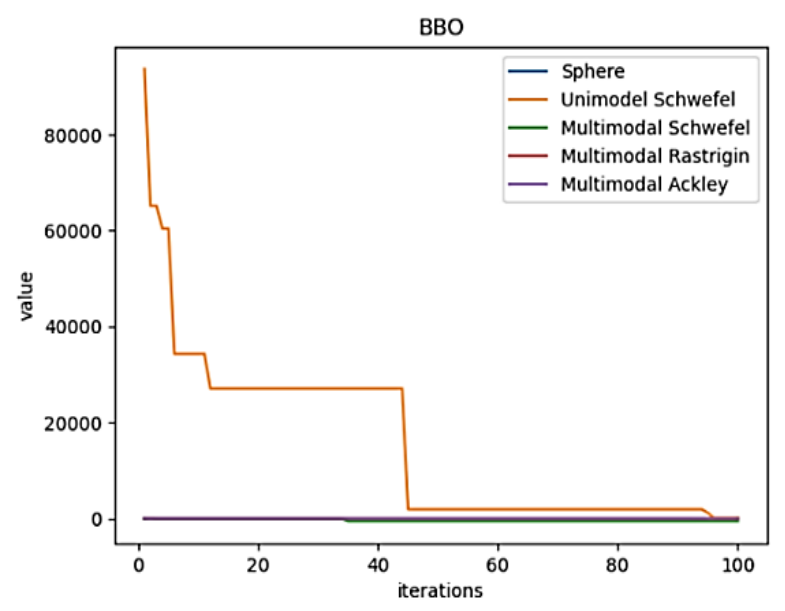

(b)

Figure 5. Plot of: (a) n-dimensional convex continuous multimodal Ackley benchmark, (b) combined benchmarks

From the figures 4 and 5, it can be observed that the BBO minimizes the benchmark functions over a certain number of iterations. Normally around the $30^{\text {th }}$ iteration mark the minima of the objective function is reached and the fitness of the solution continues to be marginally decremented until the $100^{\text {th }}$ iteration. As is the case with optimization algorithms-the main objective is to reach the local and global optima of a function as fast as possible and with as few iterations as possible. When we compare the efficacy of the BBO with other population based algorithms like the particle swarm optimization algorithm or the genetic algorithm, it has been proven that BBO is much faster and hence is the state of the art optimization algorithm. 


\section{CONCLUSION}

The presented BBO algorithm is generally in a way that is better than different strategies, or the other way around, considering the no free lunch hypothesis. Nonetheless, it might be studied and researched further to evaluate the exhibition of biogeography based optimization techniques comparative with different techniques for issues with explicit highlights. There is also a prospect of probabilistic sharing of data between species in a habitat likewise in the solutions between which a comparison paradigm can be drawn. It is inevitable that species will migrate to neighbouring places that are near to their homeland. It is in their nature. In genetic algorithms, niching also works in the same manner. BBO is an amalgamation of a handful of PB algorithms. The subtleties of the species model can always be referred to improve a given solution in terms of speed of optimization. We utilized direct, straight mu and lambda curves. However the curves will change under changing circumstances given rise to much better results. It is always assumed that habitats with basic non zero habitat suitability index provide impetus to any species that would give animal types a minimal bound that is more noteworthy than zero.

\section{ACKNOWLEDGEMENTS}

This research work was funded by “Woosong University's Academic Research Funding-2021”.

\section{REFERENCES}

[1] A. R. Wallace, "The geographical distribution of animals (two volumes)," Boston, MA: Adamant Media Corporation, vol. 2, pp. 6-10, Aug 2005.

[2] J. Vetter, "Other line: human biogeography and field practice in the eastern colonial tropics," Journal of the History of Biology volume, vol. 39, pp. 89-123, Mar. 2006, doi: 10.1007/s10739-005-6543-4.

[3] D. S. Jachowski and N. J. Singh, "Toward a mechanistic understanding of animal migration: incorporating physiological measurements in the study of animal movement," Conservation Physiology, vol. 3, no. 1, pp. 5-10, Aug. 2015, doi: 10.1093/conphys/cov035.

[4] W Edward Steinmueller, 'Will new information and communication technologies improve the 'codification' of knowledge?," Industrial and Corporate Change, vol. 9, no. 2, pp. 361-376, Jun. 2000, doi: 10.1093/icc/9.2.361.

[5] G. Wang, L. Guo, H. Duan, L. Liu, and H. Wang, "Dynamic deployment of wireless sensor networks by biogeography based optimization algorithm," Journal of Sensor and Actuator Networks, vol. 1, no. 2, pp. 86-96, Jul. 2012, doi: 10.3390/jsan1020086.

[6] M. V. Lomolino and J. H. Brown, "The reticulating phylogeny of island biogeography theory," The Quarterly Review of Biology, vol. 84, no. 4, pp. 1-9, Dec. 2009, doi: 10.1086/648123.

[7] A. Rakotomamonjy, F. Bach, S. Canu, and Yves Grandvalet, "More efficiency in multiple kernel learning," Proc. of the 24th int. conf. on Machine learning, vol. 2, pp 775-782, Jun. 2007, doi: 10.1145/1273496.1273594.

[8] D. Simon, "Biogeography-based optimization," IEEE Transactions on Evolutionary Computation, vol. 12, no. 6, pp. 702-713, Dec. 2008, doi: 10.1109/TEVC.2008.919004.

[9] D. S. Bernstein, "Optimization R Us", IEEE Control Systems Magazine, vol. 26, pp. 6-7, Mar 2006.

[10] J. M. Hoch, E. R. Sokol, A. D. Parker, J. C. Trexler, "Migration strategies vary in space, time, and among species in the small-fish metacommunity of the everglades," Copeia, vol. 1, pp. 157-169, Feb. 2015, doi: 10.1643/CE-14-040.

[11] T. Hiltunen, J. Laakso, and V. Kaitala, "Interactions between environmental variability and immigration rate control patterns of species diversity," Ecological Modelling, vol. 194, no. 1-3, pp. 125-131, Mar. 2006, doi: 10.1016/j.ecolmodel.2005.10.010.

[12] S. Rodzin and O. Rodzina, "Metaheuristics memes and biogeography for transcomputational combinatorial optimization problems," 6th Int. Conf. Cloud System and Big Data Engineering, 2016, pp. 1-5.

[13] D. M. Richardson and R. J. Whittaker, "Conservation biogeography-foundations, concepts and challenges," Diversity and Distributions, vol. 16, pp. 313-320, May 2010, doi: 10.1111/j.1472-4642.2010.00660.x.

[14] J. Franklin, "Moving beyond static species distribution models in support of conservation biogeography," Diversity and Distributions, vol. 16, no. 3, pp. 321-330, May 2010, doi: 10.1111/j.1472-4642.2010.00641.x.

[15] Anusheema Chakraborty and P K Joshi, "Simulation-based approaches for ecological niche modelling," Environmental and Agricultural Informatics, vol. 10, pp. 805-827, Mar. 2020.

[16] H. Ma, "An analysis of the equilibrium of migration models for biogeography-based optimization," Information Sciences, vol. 180, no. 18, pp. 3444-3464, Sep. 2010, doi: 10.1016/j.ins.2010.05.035.

[17] S. T. Jackson and D. F. Sax, "Balancing biodiversity in a changing environment: extinction debt, immigration credit and species turnover," Trends in Ecology and Evolution, vol. 25, no. 3, pp. 153-160, Dec. 2010.

[18] D. Simberloff, "Species turnover and equilibrium island biogeography," Science, vol. 194, no. 4265, pp. 572-578, Nov. 2020, doi: 10.1126/science.194.4265.572.

[19] D. Tilman, P. B. Reich, and J. M. H. Knops, "Biodiversity and ecosystem stability in a decade-long grassland experiment," Nature, vol. 441, pp. 629-632, Aug. 2006, doi: 10.1038/nature04742.

[20] U. Singh, H. Kumar, and T. S. Kamal, "Linear array synthesis using biogeography based optimization," Progress in Electromagnetics Research, vol. 11, pp. 25-36, Mar. 2010.

Int J Artif Intell, Vol. 10, No. 4, December 2021: 982 - 989 
[21] S. S. Reddy and C. S. Rathnam, "Optimal power flow using glowworm swarm optimization," International Journal of Electrical Power and Energy Systems, vol. 80, pp. 128-139, 2016, doi: 10.1016/j.ijepes.2016.01.036.

[22] S. Binitha and S. S. Sathya, "A survey of bio inspired optimization algorithms," International Journal of Soft Computing and Engineering (IJSE), vol. 2, no. 2, pp. 137-151, Sep. 2012.

[23] S. S. Reddy, "Optimal power flow using hybrid differential evolution and harmony search algorithm," International Journal of Machine Learning and Cybernetics, vol. 10, pp. 1077-1091, 2019, doi: 10.1007/s13042-018-0786-9.

[24] A. F. Cord et al., "Delineating probabilistic species pools in ecology and biogeography," Probabilistic Species Pools. Global Ecology and Biogeography, vol. 25, no. 4, pp. 489-501, Jan. 2016, doi: 10.1111/geb.12422.

[25] H. Bruelheide, B. Jimenez-Alfaro, U. Jandt, and F. M. Sabatini, "Deriving site-specific species pools from large databases," Ecography, vol. 43, no. 8, pp. 1215-1228, Jan. 2020, doi: 10.1111/ecog.05172.

[26] M Kaveh, M. Khishe, and M. R. Mosavi, "Design and implementation of a neighborhood search biogeographybased optimization trainer for classifying sonar dataset using multi-layer perceptron neural network," Analog Integrated Circuits and Signal Processing, vol. 100, no. 1, pp. 405-428, Mar. 2019, doi: 10.1007/s10470-018-1366-3.

[27] S. S. Reddy, "Optimal reactive power scheduling using cuckoo search algorithm," International Journal of Electrical and Computer Engineering, vol. 7, no. 5, pp. 2349-2356, Oct. 2017.

[28] D. Simon, R. Rarick, M. Ergezer, and D. Du, "Analytical and numerical comparisons of biogeography-based optimization and genetic algorithms," Information Sciences, vol. 181, no. 7, pp. 1224-1248, Apr. 2011,

[29] A. E. Gelfand, A. M. Schmidt, S. Wu, J. Silander, A. Latimer, and A. G. Rebelo, "Modelling species diversity through species level hierarchical modelling," Journal of the Royal Statistical Society: Series C (Applied Statistics), vol. 54, no. 1, pp. 1-20, Oct. 2004, doi: 10.1111/j.1467-9876.2005.00466.x.

[30] S. S. Reddy, B. K. Panigrahi, "Optimal power flow using clustered adaptive teaching learning-based optimisation," Int. Journal of Bio-Inspired Computation, vol. 9, no. 4, pp. 226-234, 2017, doi: 10.1504/IJBIC.2017.084316.

[31] M. Dorigo, M. Birattari and T. Stutzle, "Ant colony optimization," in IEEE Computational Intelligence Magazine, vol. 1, no. 4, pp. 28-39, Nov. 2006, doi: 10.1109/MCI.2006.329691.

[32] K. B. Kesur, "Advances in genetic algorithm optimization of traffic signals," Journal of Transportation Engineering, vol. 135, no. 4, pp. 160-173, Jan. 2009, doi: 10.1061/(ASCE)0733-947X(2009)135:4(160).

[33] S. S. Reddy and P. R. Bijwe, "Multi-objective optimal power flow using efficient evolutionary algorithm," International Journal of Emerging Electric Power Systems, vol. 18, no. 2, 2017, doi: 10.1515/ijeeps-2016-0233.

[34] Y Huang, "Research status and applications of nature-inspired algorithms for agri-food production," Int. Journal of Agricultural and Biological Engineering, vol. 13, no. 4, pp. 1-6, Jul. 2020, doi: 10.25165/j.ijabe.20201304.5501.

[35] R. Poli, J. Kennedy, and T. Blackwell, "Particle swarm optimisation," Swarm Intelligence, vol. 1, pp. 33-57, Jan. 2007, doi: 10.2139/ssrn.2693499. 